\title{
Correlation Inequalities and Uniqueness of the Equilibrium State for the Plane Rotator Ferromagnetic Model
}

\author{
A. Messager, S. Miracle-Sole, and C. Pfister \\ Centre de Physique Théorique, CNRS, F-13274 Marseille, France
}

\begin{abstract}
We derive new inequalities for the plane rotator ferromagnetic model and use them to obtain the following results:

1) If the model is isotropic, the derivability of the free energy as function of the magnetic field $h$ implies the existence of a unique translation invariant Gibbs state and if furthermore $h=0$ all Gibbs states are invariant by rotation of the spins.

2) If the model is anisotropic the above assertion holds for $h$ non-zero.

3) If the model is anisotropic then there are at most two extremal translation invariant Gibbs states for almost all values of the anisotropy parameter.
\end{abstract}

\section{Introduction}

In a recent work [1] Lebowitz derived new inequalities for the ferromagnetic Ising model which are very useful to obtain information about the number of pure phases that can coexist in such systems. In the following section we derive the analogous inequalities for the plane rotator ferromagnetic model and we also observe that the inequalities of Ginibre [2] can be slightly generalized. Using the fact that the translation invariant Gibbs states can be defined in terms of tangent functionals to the free energy (see $[16,3,4]$ ), we prove in Section 3 the results mentioned in the abstract. Among them the unicity of the translation invariant Gibbs state for the isotropic model at zero magnetic field was obtained by Bricmont et al. [5] quite recently. We refer the reader to the Section 4 for further comments.

\section{Inequalities}

Let $\Lambda=(1, \ldots, n)$ be a finite set of sites which we shall think as a subset of a regular $d$ dimensional lattice $\mathscr{L}$, say $\Lambda \subset \mathscr{L}=Z^{d}$. The spin at the site $i \in \Lambda$ is described by a two-dimensional unitary vector

$$
\sigma_{i}=\left(x_{i}, y_{i}\right)=\left(\cos \theta_{i}, \sin \theta_{i}\right) ; \quad \theta_{i} \in[0,2 \pi] \text {. }
$$


The set $K_{A}$ of the $\theta=\left\{\theta_{1}, \ldots, \theta_{n}\right\}$ is the set of configurations of the system. If $H_{A}(\theta)$ is the energy of the configuration $\theta \in K_{A}$ and $\beta=1 / k T$ the inverse temperature

$$
\mu_{\Lambda}(d \theta)=Z_{\Lambda}^{-1} \exp \left[-\beta H_{\Lambda}(\theta)\right] d \theta ; \quad d \theta=\prod_{i=1}^{n}\left[(1 / 2 \pi) d \theta_{i}\right]
$$

describes the Gibbs measure of the finite system defined on $\Lambda, Z_{A}$ being the corresponding partition function. The expectation value of a function $F$ defined on $K_{\Lambda}$ is

$$
\langle F\rangle_{A}=\int_{K_{A}} \mu_{\Lambda}(d \theta) F(\theta)
$$

and $-p_{A}=\frac{1}{|\Lambda|} \log Z_{\Lambda}$ is the free energy. We introduce the set $\mathfrak{M}_{\Lambda}$ of all $M=\left\{M_{1}, \ldots, M_{n}\right\}$, the $M_{i}$ taking integer values, and we write $M \theta=\sum_{i=1}^{n} M_{i} \theta_{i}$ if $M \in \mathfrak{M}_{\Lambda}$ and $\theta \in K_{\Lambda}$.

Proposition 1. Let $J$ and $J^{\prime}$ be two real-valued functions defined on $\mathfrak{M}_{A}$ and for each $M \in \mathfrak{M}_{A}$ let $\psi_{M} \in[0,2 \pi]$. Consider the two measures on $K_{A}$

$$
\begin{aligned}
\mu(d \theta) & =Z^{-1} \exp \left\{\sum_{M \in \mathfrak{M}_{A}} J(M) \cos M \theta\right\} d \theta \\
\mu^{\prime}(d \theta) & =\left(Z^{\prime}\right)^{-1} \exp \left\{\sum_{M \in \mathfrak{M}_{\Lambda}} J^{\prime}(M) \cos \left(M \theta-\psi_{M}\right)\right\} d \theta
\end{aligned}
$$

and write $\langle\rangle.\left(\right.$ resp. $\left.\langle.\rangle^{\prime}\right)$ for the corresponding expectation values. If $J(M) \geqq\left|J^{\prime}(M)\right|$ for all $M \in \mathfrak{M}_{\Lambda}$ then

$$
\begin{aligned}
& \langle\cos M \theta \cos N \theta\rangle-\left\langle\cos \left(M \theta-\varphi_{M}\right) \cos \left(N \theta-\varphi_{N}\right)\right\rangle^{\prime} \\
& \quad \geqq\left|\langle\cos M \theta\rangle\left\langle\cos \left(N \theta-\varphi_{N}\right)\right\rangle^{\prime}-\left\langle\cos \left(M \theta-\varphi_{M}\right)\right\rangle^{\prime}\langle\cos N \theta\rangle\right|
\end{aligned}
$$

for any $M, N \in \mathfrak{M}_{\Lambda}$ and any $\varphi_{M}, \varphi_{N} \in[0,2 \pi]$.

Proof. Proposition 1 is a slight improvement of a result by Ginibre [2]. It is obtained by remarking that

$$
\begin{aligned}
2\left[J(M) \cos M \theta+J^{\prime}(M) \cos \left(M \theta-\psi_{M}\right)\right] \\
=\left[J(M)+J^{\prime}(M)\right]\left[\cos M \theta+\cos \left(M \theta-\psi_{M}\right)\right] \\
\quad+\left[J(M)-J^{\prime}(M)\right]\left[\left(\cos M \theta-\cos \left(M \theta-\psi_{M}\right)\right]\right.
\end{aligned}
$$

and

$$
\begin{aligned}
& \cos M \theta+\cos \left(M \theta^{\prime}-\psi_{M}\right)=2 \cos \left(M \Phi-\psi_{M} / 2\right) \cos \left(M \Phi^{\prime}-\psi_{M} / 2\right) \\
& \cos M \theta-\cos \left(M \theta^{\prime}-\psi_{M}\right)=2 \sin \left(M \Phi-\psi_{M} / 2\right) \sin \left(M \Phi^{\prime}-\psi_{M} / 2\right)
\end{aligned}
$$

where

$$
\Phi_{i}=\frac{1}{2}\left(\theta_{i}^{\prime}+\theta_{i}\right) \quad \Phi_{i}^{\prime}=\frac{1}{2}\left(\theta_{i}^{\prime}-\theta_{i}\right)
$$


The inequality to prove is equivalent to the positivity of

$$
\begin{aligned}
& \langle\cos M \theta \cos N \theta\rangle-\left\langle\cos \left(M \theta-\varphi_{M}\right) \cos \left(N \theta-\varphi_{N}\right)\right\rangle^{\prime} \\
& \quad \pm\left[\langle\cos M \theta\rangle\left\langle\cos \left(N \theta-\varphi_{N}\right)\right\rangle^{\prime}-\left\langle\cos \left(M \theta-\varphi_{M}\right)\right\rangle^{\prime}\langle\cos N \theta\rangle\right] \\
& =\iint \mu(d \theta) \mu^{\prime}\left(d \theta^{\prime}\right)\left[\cos M \theta \mp \cos \left(M \theta^{\prime}-\varphi_{M}\right)\right]\left[\cos N \theta \pm \cos \left(N \theta^{\prime}-\varphi_{N}\right)\right] .
\end{aligned}
$$

This is proved by expanding the exponential and observing that by taking into account the identities above it can be written as a sum of terms of the form

$$
\iint d \theta d \theta^{\prime} f(\Phi) f\left(\Phi^{\prime}\right)=\left[\int d \Phi f(\Phi)\right]^{2}
$$

with positive coefficients.

As a particular case of Proposition 1 one obtains under the same hypothesis that $\langle\cos M \theta\rangle-\langle\cos (M \theta-\varphi)\rangle^{\prime} \geqq 0$ for any $M \in \mathfrak{M}_{\Lambda}$ and $\varphi \in[0,2 \pi]$.

One can also introduce purely imaginary angles $i \psi_{M}$ in the measure $\mu(d \theta)$ and obtain a generalization of Proposition 1 . We state it on a slight modificated way. Let $J_{1}, J_{2}$ and $J_{1}^{\prime}, J_{2}^{\prime}$ be two pairs of real valued functions on $\mathfrak{M}_{A}$, define

$$
\begin{aligned}
& \mu(d \theta)=Z^{-1} \exp \left\{\sum_{M \in \mathfrak{M}_{\Lambda}} J_{1}(M) \cos M \theta+i J_{2}(M) \sin M \theta\right\} d \theta \\
& \mu^{\prime}(d \theta)=\left(Z^{\prime}\right)^{-1} \exp \left\{\sum_{M \in \mathfrak{M}_{\Lambda}} J_{1}^{\prime}(M) \cos M \theta+J_{2}^{\prime}(M) \sin M \theta\right\} d \theta
\end{aligned}
$$

and assume that

$$
J_{1}(M) \geqq 0, \quad J_{1}(M)^{2}-J_{2}(M)^{2} \geqq J_{1}^{\prime}(M)^{2}+J_{2}^{\prime}(M)^{2} \text { for all } M \in \mathfrak{M}_{\Lambda} .
$$

Then the inequality of Proposition 2 is still true.

The following result is the extension of the inequalities of Lebowitz [1].

Proposition 2. Let $\mathfrak{M}_{\Lambda}^{+}$be the set of $A=\left\{A_{1}, \ldots, A_{n}\right\}$, the $A_{i}$ taking non-negative integer values. For $A \in \mathfrak{M}_{\Lambda}^{+}$and $q=\left\{q_{1}, \ldots, q_{n}\right\}, g_{i} \in \boldsymbol{R}$, write $q_{A}=\prod_{i=1}^{n} q_{i}^{A_{i}}$. Let $J_{1}, J_{2}$ and $J_{1}^{\prime}, J_{2}^{\prime}$ be two pairs of real-valued functions on $\mathfrak{M}_{\Lambda}^{+}$. Define

$$
\begin{aligned}
\mu(d \theta) & =Z^{-1} \exp \left\{\sum_{A \in \mathfrak{M}_{\perp}^{+}}\left[J_{1}(A) x_{A}+J_{2}(A) y_{A}\right]\right\} d \theta \\
\mu^{\prime}(d \theta) & =\left(Z^{\prime}\right)^{-1} \exp \left\{\sum_{A \in \mathfrak{M}_{\perp}}\left[J_{1}^{\prime}(A) x_{A}+J_{2}^{\prime}(A) y_{A}\right]\right\} d \theta
\end{aligned}
$$

to be the Gibbs measures of two systems on $\Lambda$, and assume that

$$
J_{1}(A) \geqq\left|J_{1}^{\prime}(A)\right|, J_{2}^{\prime}(A) \geqq\left|J_{2}(A)\right| \text { for all } A \in \mathfrak{M}_{A}^{+} .
$$

Then

$$
\begin{aligned}
& \left\langle x_{A}\right\rangle-\left\langle x_{A}\right\rangle^{\prime} \geqq\left|\left\langle x_{A} x_{B}\right\rangle\left\langle x_{B}\right\rangle^{\prime}-\left\langle x_{A} x_{B}\right\rangle^{\prime}\left\langle x_{B}\right\rangle\right| \\
& \left\langle y_{A}\right\rangle^{\prime}-\left\langle y_{A}\right\rangle \geqq\left|\left\langle y_{A} y_{B}\right\rangle\left\langle y_{B}\right\rangle^{\prime}-\left\langle y_{A} y_{B}\right\rangle^{\prime}\left\langle y_{B}\right\rangle\right|
\end{aligned}
$$

for any $A, B \in \mathfrak{M}_{A}^{+}$. 
Proof. It is sufficient to prove that the following quantity is positive

$$
\begin{aligned}
& \left\langle x_{A}\right\rangle-\left\langle x_{A}\right\rangle^{\prime} \pm\left[\left\langle x_{A} x_{B}\right\rangle\left\langle x_{B}\right\rangle^{\prime}-\left\langle x_{A} x_{B}\right\rangle^{\prime}\left\langle x_{B}\right\rangle\right] \\
& =\iint \mu(d \theta) \mu\left(d \theta^{\prime}\right)\left(x_{A}-x_{A}^{\prime}\right)\left(1 \pm x_{B} x_{B}^{\prime}\right) .
\end{aligned}
$$

We have a corresponding expression for the $y$ 's. Introduce the variables

$$
\begin{array}{ll}
\alpha_{i}=x_{i}+x_{i}^{\prime} & \gamma_{i}=y_{i}^{\prime}+y_{i} \\
\beta_{i}=x_{i}-x_{i}^{\prime} & \delta_{i}=y_{i}^{\prime}-y_{i}
\end{array}
$$

and notice that the binomials $x_{A}+x_{A}^{\prime}, x_{A}-x_{A}^{\prime}, y_{A}^{\prime}+y_{A}, y_{A}^{\prime}-y_{A}$, for all $A \in \mathfrak{M}_{A}^{+}$can be developped as sums of products of the variables $\alpha_{i}, \beta_{i}, \gamma_{i}, \delta_{i}$ with positive coefficients. From this it appears that Proposition 2 is a particular case of the following lemma.

Lemma 1. Let $A, B, C, D \in \mathfrak{M}_{\Lambda}^{+}$and let $g$ be a real function on $K_{\Lambda}$ such that $|g(\theta)| \leqq 1$ and $g(\theta) \cdot g\left(\theta^{\prime}\right)$ is invariant under the two symmetries $\theta_{i} \rightarrow-\theta_{i}, \theta_{i}^{\prime} \rightarrow-\theta_{i}^{\prime}$ and $\theta_{i} \rightarrow \pi-\theta_{i}$, $\theta_{i}^{\prime} \rightarrow \pi-\theta_{i}^{\prime},(i=1, \ldots, n)$. Then, under the hypothesis of Proposition 2,

$$
\iint \mu(d \theta) \mu^{\prime}\left(d \theta^{\prime}\right) \alpha_{A} \beta_{B} \gamma_{G} \delta_{D}\left[1 \pm g(\theta) g\left(\theta^{\prime}\right)\right] \geqq 0 .
$$

Proof. We write

$$
\begin{aligned}
2\left[J_{1}(A) x_{A}+J_{1}^{\prime}(A) x_{A}^{\prime}\right] & =\left[J_{1}(A)+J_{1}^{\prime}(A)\right]\left(x_{A}+x_{A}^{\prime}\right)+\left[J_{1}(A)-J_{1}^{\prime}(A)\right]\left(x_{A}-x_{A}^{\prime}\right) \\
2\left[J_{2}(A) y_{A}+J_{2}^{\prime}(A) y_{A}^{\prime}\right] & =\left[J_{2}^{\prime}(A)+J_{2}(A)\right]\left(y_{A}^{\prime}+y_{A}\right)+\left[J_{2}^{\prime}(A)-J_{2}(A)\right]\left(y_{A}^{\prime}-y_{A}\right) .
\end{aligned}
$$

Therefore, under the hypothesis of Proposition 2 , the exponential in $\mu(d \theta) \mu^{\prime}\left(d \theta^{\prime}\right)$ can be expanded as a sum of products of the variables $\alpha_{i}, \beta_{i}, \gamma_{i}, \delta_{i}$ with positive coefficients. We see, by taking into account the symmetries of the positive measure $d \theta d \theta^{\prime}\left[1 \pm g(\theta) g\left(\theta^{\prime}\right)\right]$ that the quantity $Q_{i}$ defined by

$$
\begin{aligned}
& Q_{i}\left(A_{i}, B_{i}, C_{i}, D_{i}\right)=\int d \theta_{i} d \theta_{i}^{\prime}\left(1 \pm g\left(\theta_{i}\right) g\left(\theta_{i}^{\prime}\right) \alpha_{i}^{A_{i}} \beta_{i}^{B_{i}} \gamma^{C_{i}} \delta_{i}^{D_{i}}\right. \text { satisfies } \\
& Q_{i}=(-1)^{A_{i}+B_{i}} Q_{i}=(-1)^{C_{i}+D_{i}} Q_{i}=(-1)^{B_{i}+D_{i}} Q_{i} .
\end{aligned}
$$

Hence the only terms which give a non-zero contribution to (1) are products of the $Q_{i}$ with $A_{i}, B_{i}, C_{i}, D_{i}$ all even or all odd. The lemma is thus proved since

$$
\alpha_{i} \beta_{i} \gamma_{i} \delta_{i}=\left(x_{i}^{2}-x_{i}^{\prime 2}\right)\left(y_{i}^{\prime 2}-y_{i}^{2}\right)=\left(\cos ^{2} \theta_{i}-\cos ^{2} \theta_{i}^{\prime}\right)^{2} \geqq 0 \text {. }
$$

Remarks. 1) From Lemma 1

$$
\iint \mu(d \theta) \mu^{\prime}\left(d \theta^{\prime}\right)\left(x_{A} \mp x_{A}^{\prime}\right)\left(x_{B} \pm x_{B}^{\prime}\right) \geqq 0 .
$$

Therefore under the hypothesis of Proposition 2, the inequality $\left\langle x_{A} x_{B}\right\rangle-\left\langle x_{A} x_{B}\right\rangle^{\prime}$ $\geqq\left|\left\langle x_{A}\right\rangle\left\langle x_{B}\right\rangle^{\prime}-\left\langle x_{A}\right\rangle^{\prime}\left\langle x_{B}\right\rangle\right|$, and the analogous inequality for the $y$ holds.

2) If we take $g(\theta)=\prod_{i \in A} \sin n_{i} \theta_{i} \cos m_{i} \theta_{i}$ we obtain an inequality which is also useful.

\section{Applications}

The plane rotator model or classical $X Y$ model is defined on a regular $d$-dimensional lattice $\mathscr{L}=Z^{d}$ as follows : at each site $i \in \mathscr{L}$ there is a two-dimensional vector of unit 
length, called spin,

$$
\sigma_{i}=\left(x_{i}, y_{i}\right)=\left(\cos \theta_{i}, \sin \theta_{i}\right), \quad \theta_{i} \in[0,2 \pi] .
$$

The configurations $\theta$ of the system are functions on $\mathscr{L}$ with values in $[0,2 \pi]$. $K_{\mathscr{L}}=K$ is the set of configurations on which we introduce the product topology and the corresponding Borel $\sigma$-algebra. We introduce also $\mathfrak{M}$, resp. $\mathfrak{M}^{+}$, the set of functions defined on $\mathscr{L}$ with values in $\boldsymbol{Z}$, resp. $\boldsymbol{Z}^{+}$, which have finite support (i.e. they are equal to zero except at a finite number of points). An interaction of the system is a real-valued function $J\left(\right.$.) on $\mathfrak{M}$ or a pair of real-valued functions $J_{1}(),. J_{2}($.) on $\mathfrak{M}^{+}$. Let $\psi \in K_{\mathscr{L} \backslash \Lambda}$ be a given configuration $\mathscr{L} \backslash \Lambda$ where $\Lambda$ is finite. Then the energy of the system restricted to $\Lambda$ with boundary condition $\psi$ outside $\Lambda$ is

$$
H_{\Lambda}^{\psi}(\theta)=-\sum_{\substack{M \in \mathfrak{M} \\ \operatorname{supp} M \cap A \neq \emptyset}} J(M) \cos M \theta
$$

or

$$
H_{A}^{\psi}(\theta)=-\sum_{\substack{A \in \mathfrak{M}^{+}+\\ \operatorname{supp} A \cap \Lambda \neq \emptyset}}\left[J_{1}(A) x_{A}+J_{2}(A) y_{A}\right]
$$

where the configuration $\theta \in K$ coincides with $\psi$ on $\mathscr{L} \backslash \Lambda$. In (2) and (3) we have used the notation of Section 1. Ferromagnetism corresponds to positive interactions. A probability measure on $K$ is a Gibbs measure or equilibrium state if for all finite $\Lambda$ its conditionnal probability given $\psi$ outside $\Lambda$ is equal to the Gibbs measure of the finite system on $\Lambda$ defined by the hamiltonian (2) or (3). All possible extremal Gibbs states are obtained as limits of the Gibbs measures on $\Lambda$ with various boundary conditions as $\Lambda \uparrow \mathscr{L}$ (see e.g. [4]).

We say that a Gibbs state is rotation invariant if it is invariant under a global rotation of all spins. We restrict ourselves to translation invariant interactions and make the usual conditions on the interactions in order to assure the existence of the free energy $p(J)$, resp. $p\left(J_{1}, J_{2}\right)$, which is defined as the thermodynamical limit of the free energy $p_{\Lambda}(J)$, resp. $p_{\Lambda}\left(J_{1}, J_{2}\right)$, of the finite system (see e.g. [3]).

We now analyze some consequences of Proposition 1. Consider the system defined by the hamiltonian (2) with different boundary conditions. Assume that $J(M) \geqq 0$ for all $M \in \mathfrak{M}$. Let $\langle\cos M \theta\rangle_{A}^{+}$denote the correlation functions corresponding to the boundary condition $\psi_{i}=0, i \in \mathscr{L} \backslash \Lambda$ and $\langle\cos M \theta\rangle_{\Lambda}^{(\psi)}$, those corresponding to an arbitrary boundary condition $\psi$. It easily follows from Proposition 1 that $\langle\cos M \theta\rangle_{A}^{+} \geqq\langle\cos M \theta\rangle_{A}^{(\psi)}$. Also it is easy to deduce the following facts established by Griffiths (reported in [6] and [7]) for the Ising systems: $\langle\cos M \theta\rangle_{A}^{+}$is a decreasing function of $\Lambda$ and therefore $\lim _{\Lambda \mathscr{L}}\langle\cos M \theta\rangle_{A}^{+}$ $=\langle\cos M \theta\rangle^{+}$exists, the translation invariance of this state for translation invariant interactions and finally the clustering property of this state. Moreover we have

Theorem 1. Let $J$ be a translation invariant ferromagnetic interaction (i.e. $J(M) \geqq 0$ ) defined on $\mathfrak{M}$ and consider the system associated to the hamiltonian (2). Then the correlation functions $\langle\cos M \theta\rangle^{+},\langle\sin M \theta\rangle^{+}=0, M \in \mathfrak{M}$, define a translation in- 
variant Gibbs state extremal in the convex set of all Gibbs states. Moreover if for a certain $M \in \mathfrak{M},\langle\cos M \theta\rangle^{+}=0$ then $\langle\cos M \theta\rangle=\langle\sin M \theta\rangle=0$ for all Gibbs states.

Proof. Assume that the state $\langle.\rangle^{+}$can be decomposed into other Gibbs states and let $\langle.\rangle^{\prime}$ be one of them. Since $\langle\cos M \theta\rangle^{+} \geqq\langle\cos M \theta\rangle^{\prime}$ we must have $\langle\cos M \theta\rangle^{+}$ $=\langle\cos M \theta\rangle^{\prime}$. By taking into account that $\cos M \theta, \sin M \theta$, for all $M \in \mathfrak{M}$ form a total set of functions on $K$ the extremality follows if we have $\langle\sin M \theta\rangle^{\prime}=0$. But Proposition 1 implies

$$
\begin{aligned}
\langle\cos (M \theta \pm \varphi)\rangle^{\prime} & =\langle\cos M \theta\rangle^{\prime} \cos \varphi \mp\langle\sin M \theta\rangle^{\prime} \sin \varphi \\
& =\langle\cos M \theta\rangle^{+} \cos \varphi \mp\langle\sin M \theta\rangle^{\prime} \sin \varphi \leqq\langle\cos M \theta\rangle^{+}
\end{aligned}
$$

which if $0<\varphi<\pi$ gives

$$
\pm\langle\sin M \theta\rangle^{\prime} \leqq \frac{1-\cos \varphi}{\sin \varphi}\langle\cos M \theta\rangle^{+} \underset{\varphi \rightarrow 0}{\longrightarrow} 0 .
$$

Hence $\langle\sin M \theta\rangle^{\prime}=0$ and this proves the first part of the theorem. Let now $\langle$. $>$ be an arbitrary Gibbs state. The same inequality for $\varphi=0, \pi / 2, \pi$ and $3 \pi / 2$ shows

$$
\pm\langle\cos M \theta\rangle \leqq\langle\cos M \theta\rangle^{+}, \quad \pm\langle\sin M \theta\rangle \leqq\langle\cos M \theta\rangle^{+} .
$$

This proves the second part of the theorem.

We give now sufficient conditions assuring that a Gibbs state coincides with the Gibbs state $\langle.\rangle^{+}$. We introduce first a definition. An element $M$ of $\mathfrak{M}$ or $\mathfrak{M}^{+}$is said to be even or odd according to the parity of the number $|M|=\sum_{i \in \mathscr{L}}\left|M_{i}\right|$

Lemma 2. For any Gibbs state of a system verifying the conditions of Theorem $1,\left\langle x_{A}\right\rangle$ $=\left\langle x_{A}\right\rangle^{+}$for all $A \in \mathfrak{M}^{+}$, resp. for all even $A \in \mathfrak{M}^{+}$implies $\langle\cos M \theta\rangle=\langle\cos M \theta\rangle^{+}$, $\langle\sin M \theta\rangle=0$ for all $M \in \mathfrak{M}$, resp. for all even $M \in \mathfrak{M}$.

Proof. We remark first that $\cos M \theta$ can be expanded in terms of the form $x_{A} y_{E}$ with $E$ even and if $M$ is even in terms of the form $x_{A} y_{E}$ with $A$ and $E$ even. On the other hand $x_{A}$ for all $A \in \mathfrak{M}^{+}$and $x_{E}+y_{E}$ and $x_{E}-y_{E}$ for all even $E \in \mathfrak{M}^{+}$can be written as a sum of terms of the form $\cos M \theta$ with positive coefficients. Hence, from Proposition 1 we have $\left\langle x_{A}\left(x_{E} \pm y_{E}\right)\right\rangle^{+} \geqq\left\langle x_{A}\left(x_{E} \pm y_{E}\right)\right\rangle$. Therefore if $\left\langle x_{A} x_{E}\right\rangle$ $=\left\langle x_{A} x_{E}\right\rangle^{+}$we get $\left\langle x_{A} y_{E}\right\rangle=\left\langle x_{A} y_{E}\right\rangle^{+}$for all $E$ even. This proves the part of the lemma concerning the $\langle\cos M \theta\rangle$. For $\langle\sin M \theta\rangle$ we repeat the argument of the proof of Theorem 1.

We examine now the consequence of Proposition 2.

Theorem 2. Let $J_{1}, J_{2}$ be a pair of translation invariant interactions defined on $\mathfrak{M}^{+}$ such that $J_{1}(A) \geqq 0, J_{2}(A) \geqq 0$ for all $A \in \mathfrak{M}^{+}$and consider the system defined by the hamiltonian (3). Then

1) The unicity of $\left\langle x_{i}\right\rangle, i \in \mathscr{L}$, for all translation invariant states and $\left\langle x_{i}\right\rangle \neq 0, i \in \mathscr{L}$, imply the unicity of $\left\langle x_{A}\right\rangle, A \in \mathfrak{M}^{+}$for all translation invariant states.

2) The unicity of $\left\langle x_{i} x_{j}\right\rangle$, $i$ and $j \in \mathscr{L}$, for all translation invariant states and $\left\langle x_{i} x_{j}\right\rangle \neq 0$ imply the unicity of $\left\langle x_{E}\right\rangle$ E even $\in \mathfrak{M}^{+}$, for all translation invariant states.

Proof. Let $H_{\Lambda}^{0}$ be the hamiltonian of the finite system $\Lambda$ with free boundary 
condition. Let $H_{\Lambda}^{\lambda}$ be the perturbed hamiltonian

$$
H_{\Lambda}^{\lambda}=H_{\Lambda}^{0}-\lambda \sum_{\substack{a \in G \\ \operatorname{supp}(A+a) \subset A \\ \operatorname{supp}(B+a) \subset A}} x_{A+a} x_{B+a}
$$

where $A+a$ is the translate of $A$. Let $\langle.\rangle_{A}^{\lambda}$ be the Gibbs measure of the system in $A$ defined by $H_{\Lambda}^{\lambda}$. From Proposition 2 we have if $\lambda \geqq 0$

$$
\left\langle x_{A}\right\rangle_{A}^{\lambda}-\left\langle x_{A}\right\rangle_{A}^{-\lambda} \geqq\left|\left\langle x_{A} x_{B}\right\rangle_{A}^{\lambda}\left\langle x_{B}\right\rangle_{A}^{-\lambda}-\left\langle x_{A} x_{B}\right\rangle_{A}^{-\lambda}\left\langle x_{B}\right\rangle_{A}^{\lambda}\right|
$$

and this inequality is still valid in the limit $\Lambda \uparrow \mathscr{L}$. If we assume the continuity of $\left\langle x_{A}\right\rangle^{\lambda}$ and $\left\langle x_{B}\right\rangle^{\lambda}$ at $\lambda=0$ together with $\left\langle x_{B}\right\rangle=\lim _{\lambda \rightarrow 0}\left\langle x_{B}\right\rangle^{\lambda} \neq 0$, it follows then that $\left\langle x_{A} x_{B}\right\rangle^{\lambda}$ is continuous in $\lambda$ at $\lambda=0$. Hence the infinite volume free energy per site $p(\lambda)$ is differentiable at $\lambda=0$. This implies the unicity of $\left\langle x_{A} x_{B}\right\rangle$ for the translation invariant states. Take now $A=\{i\}, B=\{j\}$. Hypothesis (1) means that $\left\langle x_{i}\right\rangle^{\lambda}$ and $\left\langle x_{j}\right\rangle^{\lambda}$ are continuous at $\lambda=0$ and that $\left\langle x_{j}\right\rangle \neq 0$, hence $\left\langle x_{i} x_{j}\right\rangle$ are unique for translation invariant states. On the other hand (from Proposition 2) $\left\langle x_{i} x_{j}\right\rangle$ $\geqq\left\langle x_{i}\right\rangle\left\langle x_{j}\right\rangle>0$. The rest of statement (1) follows by iteration of this argument. The proof of (2) is similar.

From $2\left[J_{1}(A) x_{A}+J_{2}(A) y_{A}\right]=\left[J_{1}(A)+J_{2}(A)\right]\left(x_{A}+y_{A}\right)+\left[J_{1}(A)-J_{2}(A)\right]\left(x_{A}-y_{A}\right)$ we see that the systems considered in Theorem 2 with $J_{2}(A)=0$ for all $A$ odd are particular cases of the systems of Theorem 1. They are ferromagnetic in the sense of Theorem 1 , if $J_{1}(A) \geqq\left|J_{2}(A)\right|$. For such systems, if $J_{2}(A) \geqq 0$, we can apply both Propositions 1 and 2 . We shall restrict therefore our attention to these systems.

We introduce now the notion of generating family [1]. Consider the family $\mathscr{F}$ of subsets of $\mathscr{L}$ consisting of all pairs $\{i, j\}$ of nearest neighbour points.

Lemma 3. The unicity of $\left\langle x_{i} x_{j}\right\rangle,\{i, j\} \in \mathscr{F}$ for all translation invariant states implies the unicity of $\left\langle x_{E}\right\rangle$ for all translation invariant states and all $E$ even $\in \mathfrak{M}^{+}$, if $J_{1}(A) \geqq J_{2}(A) \geqq 0, J_{2}(A)=0$ for $A$ odd and $J_{1}(\{i, j\})>0$ for all $\{i, j\} \in \mathscr{F}$.

In this case we say that the family $\mathscr{F}$ is a generating family for the even $E \in \mathfrak{M}^{+}$.

Proof. Let $\{i, j\}$ and $\{j, k\} \in \mathscr{F}$. Since $\left\langle x_{i} x_{j}\right\rangle$ and $\left\langle x_{j} x_{k}\right\rangle$ are $>0$, we see that $\left\langle x_{i} x_{j}^{2} x_{k}\right\rangle$ is unique. In particular $\left\langle x_{i} x_{j}^{2} x_{k}\right\rangle=\left\langle x_{i} x_{j}^{2} x_{k}\right\rangle^{+}$. But $x_{j}^{2}=\cos ^{2} \theta_{j}=(1 / 2)\left(1+\cos 2 \theta_{j}\right)$. Using now that $\left\langle x_{i} x_{k}\right\rangle^{+} \geqq\left\langle x_{i} x_{k}\right\rangle$ and $\left\langle x_{i} \cos 2 \theta_{j} x_{k}\right\rangle^{+} \geqq\left\langle x_{i} \cos 2 \theta_{j} x_{k}\right\rangle$ we conclude that $\left\langle x_{i} x_{k}\right\rangle=\left\langle x_{i} x_{k}\right\rangle^{+}$and $\left\langle x_{i} \cos 2 \theta_{j} x_{k}\right\rangle=\left\langle x_{i} \cos 2 \theta_{j} x_{k}\right\rangle^{+}$. The equality $\left\langle x_{i}^{2}\right\rangle=\left\langle x_{i}^{2}\right\rangle^{+}$follows by the same argument from $\left\langle x_{i}^{2} x_{j}^{2}\right\rangle=\left\langle x_{i}^{2} x_{j}^{2}\right\rangle^{+}$. We finish the proof by induction.

Remark. In the case $J_{1}(A)=J_{2}(A)=0$ for all $A$ odd $\in \mathfrak{M i}^{+}$, denote by $\langle\cos M \theta\rangle^{-}=(-1)^{|M|}\langle\cos M \theta\rangle^{+},\langle\sin M \theta\rangle^{-}=0$ the state obtained from the boundary conditions $\psi_{i}=\pi, i \in \mathscr{L} / \Lambda$. Then from Proposition 2

$$
\left\langle x_{i}\right\rangle^{+}-\left\langle x_{i}\right\rangle^{-}=2\left\langle x_{i}\right\rangle^{+} \geqq\left|\left\langle x_{A}\right\rangle^{+}\left\langle x_{A} x_{i}\right\rangle^{-}-\left\langle x_{A}\right\rangle^{-}\left\langle x_{A} x_{i}\right\rangle^{+}\right|=2\left\langle x_{A} x_{i}\right\rangle^{+}\left\langle x_{A}\right\rangle^{+} .
$$

for all even $A \in \mathfrak{M i}^{+}$. If one has $\left\langle x_{i}\right\rangle^{+}=0$ for all $i \in \mathscr{L}$ one gets that if $A$ is odd also $\left\langle x_{A}\right\rangle^{+}=0$, and from Theorem 1 that $\left\langle x_{A}\right\rangle=0$ for any Gibbs state. 
Theorem 3. Let $J$ be a translation invariant two-body interaction such that $J(\{i, j\}) \geqq 0$ and strict positive for all $\{i, j\} \in \mathscr{F}$. Let $h \geqq 0$ be the external magnetic field and $\gamma \geqq 1$ an anisotropy parameter. Consider the system associated to the hamiltonian

$$
H_{\Lambda}(\theta)=-\sum_{\substack{A \in \mathfrak{M}^{+} \\ \text {supp } A \subset A}}\left\{\gamma J(A) x_{A}+J(A) y_{A}\right\}-h \sum_{i \in \Lambda} x_{i}
$$

and let $p(\beta, \gamma, h)$ be the corresponding infinite volume free energy.

1) If $h \neq 0$ and $\partial p / \partial h$ exists (in this case $\partial p / \partial h>0$ ), there is a unique translation invariant Gibbs state.

2) If $h=0, \gamma=1$, and $\partial p / \partial h$ exists (then $\partial p / \partial h=0$ ) there is a unique translation invariant Gibbs state. Moreover all possible Gibbs states are rotation invariant.

3) If $h=0, \gamma>1$, and $\partial p / \partial \gamma$ exists, then $\langle\cos M \theta\rangle$ is unique for all translation invariant states, for all $M$ even $\in \mathfrak{M}$. Therefore any translation invariant Gibbs state is a convex linear combination of (at most) two extremal states.

Proof. Notice first that the system considered in this theorem verifies the conditions of Theorems 1 and 2.

To prove statement 1 ) we apply Theorem 2 with $\left\langle x_{i}\right\rangle=\partial p / \partial h$ and deduce that the translation invariant $\left\langle x_{A}\right\rangle$ are unique and therefore that $\left\langle x_{A}\right\rangle=\left\langle x_{A}\right\rangle^{+}$for all $A \in \mathfrak{M}$. From Lemma 2 it follows then that $\langle\cos M \theta\rangle=\langle\cos M \theta\rangle^{+},\langle\sin M \theta\rangle=0$ for all $M \in \mathfrak{M}$ and the unicity of the invariant Gibbs state is proved. It is clear that statement 1 ) is also true for any interactions $J_{1} \geqq 0, J_{2}$ with support in the even elements of $\mathfrak{M}^{+}$, and $J_{1} \geqq J_{2} \geqq 0$ in the hamiltonian (3).

To prove statement 2) we first apply the inequality $\left\langle\left(x_{i}+y_{i}\right)\left(x_{j}-y_{j}\right)\right\rangle^{+}$ $\leqq\left\langle x_{i}+y_{i}\right\rangle^{+}\left\langle x_{i}-y_{i}\right\rangle^{+}$which is found in [8], Proposition 6, (it easily follows by doing the change of variables $x_{i}^{\prime}=x_{i}+y_{i} y_{i}^{\prime}=x_{i}-y_{i}$.) Since $\left\langle x_{i}\right\rangle^{+}=\left\langle y_{i}\right\rangle^{+}=0$ we obtain $\left\langle\cos \left(\theta_{i}+\theta_{j}\right\rangle^{+}=\left\langle x_{i} x_{j}-y_{i} y_{j}\right\rangle^{+}=0\right.$. From Theorem $1\left\langle\cos \left(\theta_{i}+\theta_{j}\right)\right\rangle=0$ in all Gibbs states. In particular $\lim _{\lambda \rightarrow 0}\left\langle x_{i} x_{j}-y_{i} y_{j}\right\rangle^{ \pm \lambda}=0$ where the notations of Theorem 2 are used and $H_{A}^{\lambda}$ denotes the perturbed hamiltonian with the term $\lambda \sum_{\substack{a \in \mathscr{C} \\ i+a, j+a \in \Lambda}} x_{i+a} x_{j+a}$. Therefore

$$
\lim _{\substack{\lambda \rightarrow 0 \\ \lambda>0}}\left[\left(\left\langle x_{i} x_{j}\right\rangle^{\lambda}-\left\langle x_{i} x_{j}\right\rangle^{-\lambda}\right)+\left(\left\langle y_{i} y_{j}\right\rangle^{-\lambda}-\left\langle y_{i} y_{j}\right\rangle^{\lambda}\right)\right]=0 .
$$

Since, from Proposition 2, the two terms in the right hand side are positive, both tend to zero. This shows that $\left\langle x_{i} x_{j}\right\rangle^{\lambda}$ is continuous in $\lambda$ at $\lambda=0$ and hence that the translation invariant $\left\langle x_{i} x_{j}\right\rangle$ are unique. Moreover $\left\langle x_{i} x_{j}\right\rangle^{+}>0$. Then from Theorem 2 the translation invariant $\left\langle x_{A}\right\rangle$ for all even $A$ are unique. On the other hand, since $\left\langle x_{i}\right\rangle^{+}=0,\left\langle x_{A}\right\rangle=0$ for all odd $A$. Lemma 2 allows us to conclude then at the unicity of the translation invariant Gibbs state. The Gibbs state obtained with free boundary condition is translation invariant and spin rotation invariant and it coincides with the state $\langle.\rangle^{+}$. Hence $\langle\cos M \theta\rangle^{+}=0$ for all $M$ such that $\sum_{i \in \mathscr{L}} M_{i} \neq 0$. Theorem 1 implies then that $\langle\cos M \theta\rangle=\langle\sin M \theta\rangle=0$ for all Gibbs states if $\Gamma M: \neq 0$. i.e. that all Gibbs states are rotation invariant. 
To prove statement 3) notice that for any perturbed hamiltonian of the type considered in Theorem 2 one has $\left\langle x_{A}\right\rangle^{\lambda} \geqq\left\langle x_{A}\right\rangle^{-\lambda}$ if $\lambda \geqq 0$ and that $\lim _{\lambda \rightarrow 0}\left\langle x_{A}\right\rangle^{ \pm \lambda}$ define equilibrium correlations at $\lambda=0$. From the existence of $\partial p / \partial \gamma$ we deduce that

$$
\lim _{\substack{\lambda \rightarrow 0 \\ \lambda>0}} \sum_{\substack{A \in \mathfrak{M}^{+} \\ 0 \in \mathrm{Supp}^{+}}} J(A)\left(\left\langle x_{A}\right\rangle^{\lambda}-\left\langle x_{A}\right\rangle^{-\lambda}\right)=\partial p / \partial \gamma-\partial p / \partial \gamma=0 .
$$

Since the identity above contains only positive terms $\lim _{\lambda \rightarrow 0}\left(\left\langle x_{A}\right\rangle^{\lambda}-\left\langle x_{A}\right\rangle^{-\lambda}\right)=0$ for all $A$ such that $J(A)>0$. Hence the translation invariant $\left\langle x_{A}\right\rangle$ are unique if $J(A)>0$. On the other hand they are strictly positive. If they form a generating family for the even elements of $\mathfrak{M}^{+}$we deduce from Theorem 2 the unicity of the translation invariant $\langle\cos M \theta\rangle$ for all even $M \in \mathfrak{M}$. From this we have

$$
\begin{aligned}
\frac{1}{2}\langle\cos M \theta\rangle+\frac{1}{2}(-1)^{|M|}\langle\cos M \theta\rangle & =\frac{1}{2}\langle\cos M \theta\rangle^{+}+\frac{1}{2}\langle\cos M \theta\rangle^{-} \\
\frac{1}{2}\langle\sin M \theta\rangle+\frac{1}{2}(-1)^{|M|}\langle\sin M \theta\rangle & =0
\end{aligned}
$$

for all $M$ and all translation invariant Gibbs states. Take into account that $(-1)^{|M|}\langle$.$\rangle is again a Gibbs state (obtained by changing \psi_{i} \rightarrow \pi-\psi_{i}$ on the boundary) and apply Theorem 1 . These identities and the Choquet simplex structure of the set of Gibbs states show that if $\langle$.$\rangle is supposed to be an extremal$ invariant state it has to coincide with $\langle.\rangle^{+}$or $\langle.\rangle^{-}$. Therefore any translation invariant Gibbs state has the form

$$
\langle\cos M \theta\rangle=\lambda\langle\cos M \theta\rangle^{+}+(1-\lambda)\langle\cos M \theta\rangle^{-},\langle\sin M \theta\rangle=0
$$

for some $\lambda \in[0,1]$. It is clear that 3$)$ is also true if $J(A)$ has support on the even $A \in \mathfrak{M}^{+}$.

Corollary. Under the hypothesis of Theorem 3, for any boundary condition of the form $\psi=\left\{\psi_{i}\right\}, \psi_{i} \in[-\pi / 2, \pi / 2], \lim _{\Lambda \mp \mathscr{L}}\langle\cos M \theta\rangle^{+}=\langle\cos M \theta\rangle^{+}$and $\lim _{\boldsymbol{A T \mathscr { L }}}\langle\sin M \theta\rangle_{A}^{(\psi)}=0$ exist independently of the boundary condition, for all $M \in \mathfrak{M}$ in Cases 1) and 2) and for all even $M \in \mathfrak{M}$ in Case 3).

Proof. To these boundary conditions Proposition 2 can be applied and we have $\left\langle x_{A}\right\rangle_{A}^{+} \geqq\left\langle x_{A}\right\rangle_{A}^{(\psi)} \geqq\left\langle x_{A}\right\rangle_{A}^{(\pi / 2)},(\pi / 2)$ being the boundary condition $\psi_{i}=\pi / 2$ for all $i \in \mathscr{L} \backslash \Lambda$. Since $\lim _{\Lambda \uparrow \mathscr{L}}\left\langle x_{A}\right\rangle_{A}^{+}=\lim _{\Lambda \mp \mathscr{L}}\left\langle x_{A}\right\rangle_{A}^{(\pi / 2)}=\left\langle x_{A}\right\rangle^{+}$the corollary follows by applying Lemma 2. (Use the fact that $\left\langle x_{A}\right\rangle_{A}^{(\pi / 2)}$ is increasing in $\Lambda$ and is a translation invariant state.)

\section{Conclusion}

We consider in this section models with two-body interactions and with or without magnetic field $h$. If $h \neq 0$ there is a unique translation invariant Gibbs state if and 
only if the free energy is differentiable in $h$. By the Lee Yang circle theorem generalized to the plane rotator model [9] we know the existence of the derivative for such $h$.

At $h=0$ the equivalence above.was proved first by Bricmont et al. [5] for the isotropic model. We have presented again a brief proof of this fact based on our previous results. We have thus unicity of the translation invariant Gibbs state as soon as the spontaneous magnetization is zero, i.e. for all $\beta<\beta_{c}$. Moreover we have proved that in this case all Gibbs states are rotation invariant. So we recover for the plane rotator model the general result by Dobrushin and Schlosman [11] about the absence of broken symmetry in two-dimensional systems for all interactions decaying at infinity at least like $r^{-4}[10]$. We should expect also for anisotropic system at $h=0$ that the translation invariant Gibbs state is unique when the spontaneous magnetization is zero. This fact is true for Ising systems with two-body interactions $[1,13]$. In any case the existence of $\partial p / \partial \gamma$ implies the unicity of the translation invariant state for all $\beta<\beta_{c}$, the inverse critical temperature, since $\left\langle x_{i}\right\rangle^{+}=0$. Moreover $\partial p / \partial \gamma$ exists if and only if $\partial p / \partial \beta$ exists. (If $\partial p / \partial \beta$ exists and $\gamma>1$ then $\left\langle\cos \theta_{i} \pm \theta_{j}\right\rangle$ are unique and so $\left\langle x_{i} x_{j}\right\rangle$ for all translation invariant states.) Using integral equations of the Kirkwood-Salsburg type we have complex analyticity of the free energy in $\beta$ and $h$ at high enough $h$. If the temperature is such that the set of zeros in the Lee-Yang circle theorem does not have $z=\exp (\beta h)=1$ as accumulation point we have also real analyticity in $\beta$ at $h=0$ by a theorem of Lebowitz and Penrose [15]. So at high enough temperature there is a unique translation invariant Gibbs state.

Consider now an isotropic system in 3 or more dimensions at $h=0$ in the phase coexistence region [14]. For the translation invariant Gibbs states, it is very tempting to believe that the unicity of the expectation values of $\left\langle\cos \left(\theta_{i}-\theta_{j}\right)\right\rangle$ for all $i, j \in \mathscr{L}$ implies the unicity of $\langle\cos M \theta\rangle$ for all observables which can be generated from the previous one, in general all the spin rotation invariant correlations. From the existence of $\partial p / \partial \beta$ and the Choquet simplex structure of the set of Gibbs states one could deduce in this case that any translation invariant Gibbs state is a convex superposition of the pure phases associated to the values of an angle $\varphi \in[0,2 \pi]$ and obtained from the boundary conditions $\psi_{i}=\varphi, i \in \mathscr{L} \backslash \Lambda$. However we are not able to solve this problem with the inequalities of Section 2.

Let us finally remark that we have for simplicity restrict our attention to the plane rotator systems. More general two-dimensional vector spin systems as those considered in [5] could be treated by the same method. The same results obtained above hold also for the discrete plane vector systems in which the one point configurations are restricted to the values $\theta_{i}=k_{i} / 2 N \pi, k_{i}=0,1, \ldots, N-1$ where $N$ is some integer. In fact Proposition 1 for this system can be obtained by introducing an external interaction $K \cos N \theta_{i}$ at all $i \in \mathscr{L}$ and letting $K \rightarrow+\infty$. Proposition 2 is more general. We have discussed the one point measure $d \theta_{i}=\delta\left(x_{i}^{2}+y_{i}^{2}-1\right) d x_{i} d y_{i}$ but any measure symmetric with respect to the two axis of the form $\mu\left(d \theta_{i}\right)$ $=f\left(x_{i}, y_{i}\right) \delta\left[g\left(x_{i}^{2}\right)+y_{i}^{2}\right] d x_{i} d y_{i}$ with $f \geqq 0$ and $g$ a non-decreasing function of $x_{i}^{2}$ can as well be considered. One has then the same symmetries and the fact that $\alpha_{i} \beta_{i} \gamma_{i} \delta_{i}=\left(x_{i}^{2}\right.$ $\left.-x_{i}^{\prime 2}\right)\left(y_{i}^{\prime 2}-y_{i}^{2}\right)=\left(x_{i}^{2}-x_{i}^{\prime 2}\right)\left[g\left(x_{i}^{2}\right)-g\left(x_{i}^{\prime 2}\right)\right] \geqq 0$.

Acknowledgements. It is a pleasure to thank J. L. Lebowitz for valuable discussions. One of us (C.P.) thanks R. Stora for his kind hospitality at the Centre de Physique Théorique, CNRS Marseille. 


\section{References}

1. Lebowitz,J.L.: Coexistence of phases in ising ferromagnets. I.H.E.S. Preprint. Bures-sur-Yvette, France 1976

2. Ginibre,J.: Commun. math. Phys. 16, 310 (1970)

3. Ruelle,D.: Statistical mechanics. New York: Benjamin 1969

4. Lanford III,O.E.: In: Statistical mechanics and mathematical problems (ed. A. Lenard). BerlinHeidelberg-New York: Springer 1973

5. Bricmont,J., Fontaine,J.R., Landau,L.J.: On the uniqueness of the equilibrium state in plane rotators. U.C.L.-I.P.T. Preprint. Louvain-la-Neuve, Belgium 1977

6. Griffiths, R. B.: In: Phase transitions and critical phenomena, Vol. 1 (eds. C. Domb, M. S. Green). London: Academic Press 1972

7. Gallavotti,G., Martin-Löf,A., Miracle-Sole,S.: In : Statistical mechanics and mathematical problems (ed. A. Lenard). Berlin-Heidelberg-New York: Springer 1973

8. Kunz,H., Pfister,C.E., Vuillermot,P. A. : J. Phys. A., Math. Gen. 9, 1673 (1976)

9. Dunlop, F., Newman,C.M.: Commun. math. phys. 44, 223 (1975)

10. Mermin, N.D.: J. Math. Phys. 8, 1061 (1967)

11. Dobrushin, R.L., Schlosman,S. B.: Commun. math. Phys. 42, 31 (1975)

12. Malishev, V.A.: Commun. math. Phys. 40, 75 (1975)

13. Lebowitz,J.L., Martin-Löf, A.: Commun. math. Phys. 25, 276 (1972)

14. Frohlich,J., Simon, B., Spencer,T.: Commun. math. Phys. 50, 79 (1976)

15. Lebowitz,J.L., Penrose,O.: Commun. math. Phys. 11, 99 (1968)

16. Fisher,M.E.: J. Math. Phys. 6, 1642 (1965)

Communicated by E. Lieb

Received August 23, 1977; in revised form October 17, 1977

Note Added in Proof: In the phase coexistence region of the anisotropic model [12] all translation invariant equilibrium states are convex combinations of two extremal states as soon as the spontaneous magnetization is continuous in the temperature. 
